\title{
Systematisch-theologische Anfragen an den Islam
}

Systematisch-theologische Anfragen an den koranischen Islam können nicht einfach darin bestehen, positive christliche Glaubensaussagen geltend $\mathrm{zu}$ machen. ${ }^{1}$ Statt solcher „dogmatischen“ Kontrastierung will ich versuchen, vergleichend bestimmte Fragen in Argumentationszusammenhänge zu stellen. Ein Hauptgesichtspunkt bei einem solchen systematischen Vergleich betrifft die Frage, wie sich, sowohl im Christentum wie im Islam, die Gegenstände des religiösen Bewusstseins (z. B. also der Gottesgedanke) in diesem religiösen Bewusstsein selber (z. B. also in der Frömmigkeit bzw. dem Glauben) reflektieren - ob also Inhalt und Form der Religion einander entsprechen (bzw. wie sie es tun) oder nicht. Statt nur eine Behauptung gegen eine andere zu stellen, soll versucht werden, den Vergleich religions-logisch zu halten.

Ich habe also weder moralische oder politische Einschätzungen im Sinn, noch gar Urteile über die Frömmigkeit einzelner Muslime oder islamischer Gruppen in ihrer Vielfalt. ${ }^{2}$ Daher ist es vielleicht nützlich, schon vorweg $\mathrm{zu}$ betonen, dass meine hier vorzutragenden Überlegungen lange vor dem 11. September 2001 und aufgrund rein religionstheoretischer Einsichten sowie durch eine erschöpfende Koran-Lektüre zustande gekommen sind.

Ich konzentriere mich wegen der mir zubemessenen knappen Zeit im Folgenden auf das Gottesverhältnis bzw. die Frage nach dem einen Gott.

Bekanntlich versteht sich der Islam als reiner Monotheismus; aber auch die christliche Theologie behauptet seit ihren Anfängen (und im Anschluss an das Alte Testament), nichts anderes als den einen Gott, den Schöpfer des Himmels und der Erde, zu denken, seine Einheit aber als Dreieinigkeit. Damit ist die religiös zentrale Frage im Vergleich beider Religionen gestellt.

In vielen Suren des Koran wird diese unantastbare und unüberbietbare Einheit des ewigen Gottes - sozusagen als das religiöse Grundaxiom des Islam eingeschärft, so z. B. Sure 112, 1-4:

\footnotetext{
1 Theologische Anfragen an eine andere Religion sollten immer auch kritische Selbstanfragen an die eigene, christliche Religion sein. Dies auszuführen, ist hier kein Raum. Vgl. aber die folgende Anm.

2 Aus demselben Grund gehe ich in der Konzentration auf meinen systematischen Gesichtspunkt hier auch nicht auf die Gewalttaten in der Kirchengeschichte ein. Es gibt christlich aber vom Neuen Testament her und der Sache nach - Kriterien, um sie als unchristlichen Missbrauch der Religion zu kritisieren. Vgl. jedoch $u$. Anm. 11 .
} 
Sprich: Er ist der eine Gott, der ewige Gott; Er zeugt nicht und wird nicht gezeugt; Und keiner ist ihm gleich. ${ }^{3}$

Im Blick auf diese und viele ähnliche Aussagen ist festzuhalten: Allah ist exklusiv der schlechthin Singuläre, der in unbedingter Selbstgleichheit ist, was er ist $t^{4}$. In ihm selber gibt es weder irgendeine Differenz noch gar irgendein genetisches Verhältnis; er ist in reiner Identität nur er selbst und daher unvergleichbar mit allem anderen (d.h. geschöpflichen) Seienden: der Herr und eindeutige Herrscher, der als Einer absolut über allem thront. ${ }^{5}$

Mit diesem Gottesgedanken verbindet sich in zahlreichen Suren, wie schon anklang, die entschiedene Abwehr des (nur vage erfassten) christlichen Gedankens von der Dreieinigkeit Gottes:

So glaubet an Allah und an seinen Gesandten ${ }^{6}$ und sprechet nicht: ,Drei ${ }^{67}$... Allah ist nur ein einiger Gott: Preis ihm, dass ihm [nicht etwa] sein sollte ein Sohn! . ${ }^{8}$

Aus dieser Kritik an der Dreieinigkeit ergibt sich die systematische Sachfrage nach dem im Koran wie in der christlichen Theologie gemeinten Begriff von Einheit (als Einheit Gottes).

Es fällt auf, mit welcher positivistischen Umstandslosigkeit das Einheitsaxiom im Koran behauptet wird. Will man das leitende Verständnis von Einheit Gottes, seiner Einzigkeit und seinem Mit-sich-eins-Sein weiter präzisieren, so stellt sich einerseits die Frage, ob man nicht Gottes Einssein nach außen - d.h. im Verhältnis zur Schöpfung -, das auch der christliche Glaube für Gott unverbrüchlich festhält und darin mit dem Alten Testament und dem Koran einig ist,

3 Der Koran wird hier zitiert nach der Reclam-Ausgabe (Nr. 4206-10/10a), übersetzt von M. Henning (1960). Vgl. auch: „Bezeugt hat Allah, dass es keinen Gott gibt außer ihm ... Es gibt keinen Gott außer ihm, dem Mächtigen, dem Weisen“ (Sure 3, 16) sowie Sure 2, 158 u. 256; 3, 1 u. 16; 5, $169 ; 64,13$.

4 Sure 89, 2: „einfach“.

5 Vgl. dazu die ungemein umsichtig-ausgewogene Darstellung bei J. Baur: Der christliche Gottesglaube angesichts der Herausforderung durch den Islam (1992); in: Ders., Einsicht und Glaube. Band 2 (1994); hier: 159 u. 162.

$6 \mathrm{Ihm}$ wird auch Jesus zugeordnet.

7 D.h. seid keine Tritheisten.

8 Sure 4, 169. Sure 5, 77: „Wahrlich, ungläubig sind, die da sprechen: `Siehe, Allah ist ein dritter von drei`. Aber es gibt keinen Gott außer Allah“. Hier findet sich die Verwerfung der Annahme, dass Gott ein „Kind“ habe (5, 76), wie ebenso Sure 2, 110; 5, 116 u. ö. Vgl. Sure 6, 1.14.22; 7, 100f; 13, 17; 14, 35; 16, 53; 41, 8. Vgl. auch T. Nagel: Das Christentum im Urteil des Islam (2007), (Bursfelder Universitätsreden 24). 
unterscheiden muss von der internen Verfassung dieser Einheit in Gott bzw. für Gott selbst. ${ }^{9}$

Andererseits richtet sich eine Frage auf die logische Verfassung der koranischen Einheit selber. Nicht nur entspricht ihr ein abstraktes Jenseits, das die Vielheit alles Anderen von sich nur fernhält, sondern sie ist - und das ist hier entscheidend - eine in sich bestimmungslose Einheit, sozusagen ein ewiges Faktum, bei dem begrifflich unentscheidbar ist, ob sie sich nur einer externen Zuschreibung verdankt, d. h. Gott sozusagen nur „angetan“ wird. Danach fände sich Allah in seiner Identität gleichsam nur unmittelbar vor. Das ist deswegen wichtig, weil eine faktische, bloß abstrakte Einheit als letztes formales Einheitsprinzip logisch leer ist und weil sie ausschließlich als reine Negation von Differenz ist, was sie ist. Dieser Eine ist so mit sich eins, dass er alles Andere (bzw. alles, was nicht er selbst oder was er selbst nicht ist) von sich ausschließt. Eine über abstrakte Negation von Unterschieden (Unterschiedenheit) definierte Einheit ist, logisch gesehen, das Prinzip von Herrschaft; es impliziert tendenziell Züge von Gewalt, weil es sich durch Ausschluss alles Nicht-Identischen konstituiert. ${ }^{10}$

Von hier aus kann man nicht sagen, dass der Dschihad (strukturell) nicht zum koranischen Islam gehöre. ${ }^{11}$ Es ist unübersehbar, dass im Koran der „Weg Gottes“ selber als Kampf gegen die Ungläubigen definiert wird, wie viele Suren bezeugen. ${ }^{12}$ Damit ist eine kriegerische Ausbreitung des Islam kompatibel und ausdrücklich die Erwartung eines „Endsieges“ über die anderen Religionen verbunden. ${ }^{13}$

Zurück zur Frage des Begriffs von Einheit. Der christliche Gottesgedanke ist - weit entfernt der Nietzschesche „Monotonotheismus“ zu sein - von dem logischen Gedanken einer in sich selbst gründenden Einheit her gedacht, die sich zu sich selbst bestimmt und so einen Unterschied zu sich selber einschließt bzw. übergreift. Sie ist keine Einheit, die von Gott nur prädiziert würde - nämlich in dem bloß negativen Gedanken einer Nicht-Vielheit an Gott -, sondern sie ist eine Einheit, die aus Gott selber begriffen werden kann: als selbstmächtige und so an ihr selber wahre Einheit. Nach diesem Begriff bestimmt sich Gott selbst zu seiner

9 Das hat gegen den Islam bereits Luther getan; vgl. Weimarer Ausgabe, Band 49, 238.

10 Bezeichnend ist die negative Verfassung des Begriffs von islam (bzw. aslama): „(sich) Weggeben“ (s.u. Anm. 35). Was die Herrschaft betrifft, so hätte Hegel mit mehr Recht als das Judentum (vgl. Werke in zwanzig Bänden, 17, 80ff) den Islam als die erhabene Religion von Herr und Knecht beschreiben können (s.u. Anm. 36). Zu dessen „Fanatismus“ vgl. a.a.O. 73 u. 86; zum Islam überhaupt (weltgeschichtlich) Band 12, 428ff.

$11 \mathrm{Vgl}$. insbes. Sure 61. Jedenfalls kann der Dschihad aus dem Koran legitimiert werden, mit dem Neuen Testament Ähnliches aber nicht.

12 Sure 4, 76.98; 9, 20.38.41.112; 22, 77; 49, 15; 57, 10; 60, 1; 61, 11; 73, 20.

13 Sure 8,$40 ; 9,33 ; 61,90$. 
Einheit, und so ist sie Gottes eigene Einheit mit sich: selbsthaft statt unmittelbare Selbstgleichheit.

Der christliche Begriff von Gott als dem Dreieinigen stellt sich somit logisch als eine gedankliche Alternative zur unmittelbaren Einheitsbehauptung des Koran dar. Der dreieinige Gott kann als der wahrhaft Eine gedacht werden, weil er sich aus sich und durch sich ( $a$ se et per se) selber zu seiner Einheit bestimmt; sie ist daher als konkrete und lebendige Einheit zu denken. ${ }^{14}$

Die religiösen Folgen dieses christlichen Einheitskonzeptes sind erheblich und durchgreifend.

1. Zunächst folgt aus dem trinitarischen Gedanken die Frage an den Islam, ob hier eine logisch wahre Einheit überhaupt gedacht werden kann oder „Einheit“ nicht unterbestimmt ist. ${ }^{15}$ Im Unterschied dazu ist gerade der Begriff des dreieinigen Gottes als Inbegriff wahrer Einheit (sc. aus sich selber und für sich selber) zu verstehen, wie man bei so verschiedenen Denkern wie Luther, ${ }^{16}$ Schelling, ${ }^{17}$ K. Barth ${ }^{18}$ oder W. Pannenberg ${ }^{19}$ nachlesen kann.

2. Die trinitarisch gedachte Einheit ist lebendige Einheit, weil sie in sich Unterschiede zulässt und ihr Anderes zu integrieren vermag. ${ }^{20}$ So wird die Offenheit Gottes für die Schöpfung einer endlichen Welt, ${ }^{21}$ für sein Sich-

14 Wie das mit den drei innergöttlichen Instanzen (traditionell: „,Vater“, „Sohn“ und H.Geist) zusammenhängt, die in ihrem perichoretischen Ineinandersein die göttliche Einheit konstituieren, muss hier nicht entfaltet werden. Nachvollziehbar dürfte sein, dass die Tradition Drei-Einigkeit irgendwie als innergöttliche Selbstdifferenzierung begriffen hat.

15 Solange diese Frage offen bleibt, sind die unmittelbaren, kämpferischen Wahrheitsansprüche im Koran (vgl. Sure 2, 171; 5, 52; 6, 73; 9, 33; 22, 53; 23, 64; 24, 25; 39, 34; 48, 28; 61, 9) mit Gründen nicht zu entscheiden.

16 „fatemur fester unum deum esse, quam Gentiles“ (Weimarer Ausgabe, Band 49, 238,7).

17 Zur Trinität als dem wahrem Monotheismus vgl. Philosophie der Mythologie, 2. Band (ND Darmstadt 1966), 79 und Philosophie der Offenbarung, 1. Band (1966), 316f u. 338.

18 ,Von diesem Wesen Gottes nun ist zu sagen, ... dass vielmehr gerade in der Dreiheit der `Personen` seine Einheit besteht“ (Die Kirchliche Dogmatik, Band I/1, 396; vgl. 371). Der Terminus „Person“ ist hier uneigentlich zu nehmen.

19 „dass der Gedanke der Einheit Gottes ohne Trinität gar nicht sachgemäß und konsistent gedacht werden kann“ (Systematische Theologie, Band I (1988), 317). Zum „konkreten Monotheismus“ vgl. a.a.0. 363 mit A. 220).

20 Luther kann sogar formulieren: „dum se multiplicat, maxime idem manet et fit“: Indem er sich [in sich] vervielfältigt, bleibt er und wird er auf absolute Weise Derselbe (Weimarer Ausgabe, Band 1, 27,18).

21 Es scheint fraglich, ob sich Allahs Eins-Sein so eindeutig an der Schöpfung ablesen lässt, wie der Koran behauptet. Weist die geschaffene Wirklichkeit nicht eher vestigia trinitatis auf, wie die Theologie seit alters darlegt? Ist nicht allein Gottes Geist-Sein Bedingung der Möglichkeit, die Schöpfung des Menschen (als eines der Schöpfung bewussten Wesens (imago Dei) und eines zur Gemeinschaft von Mann und Frau bestimmten Wesens (vgl. 1Mose 1, 27)) zu begreifen? Wird Gott 
Offenbaren und insbesondere für seine christlich verstandene Menschwerdung aus Gottes innerstem Wesen heraus nachvollziehbar. Besonders die Menschwerdung, d.h. Gottes eigenes Sein in einem wehrlosen Menschen (Jesus von Nazareth) bedeutet den Bruch mit der Unmittelbarkeit abstrakter Identität. Was dieses Thema angeht, so zeigt der Koran in der endlosen, aber als biologischer Vorgang missverstandenen These, Gott habe keinen Sohn „gezeugt“22, nirgendwo wirkliche Kenntnis der christlichen Trinitätslehre, sondern hat die Inkarnation geist-los missverstanden.

Bei dem bewegungslos Einen kann es keine Selbstunterscheidung Gottes von seinem ewigen Logos noch seine eigene Entäußerung in einen Menschen geben, d.h. keinen inkarnatorischen Selbsteinsatz Gottes, der sich als die hingebende Liebe selber unser Leiden zu eigen macht und unseren Widerspruch zu ihm von sich her überwindet, also keinen Herrn der Gnade, „der arm wurde um unsertwillen, damit wir durch seine Armut reich würden“ (2Kor 8, 9). Noch weniger kann es eine Solidarität Gottes mit dem gekreuzigten Sohn geben. Der Koran leugnet konsequent die Kreuzigung Jesu (Sure 4, 156) und behauptet seine schemenhafte Erhöhung zu Gott (Sure 4, 156; 3, 48).

So wird Jesus zwar (wie frühere Propheten) zu einem „Gesandten“ Allahs erklärt, ${ }^{23}$ Mohammed aber als der alles vollendende, letzte und höchste Gesandte, ${ }^{24}$ als „das Siegel der Prophetie“ behauptet ${ }^{25}$ - ohne dass diese Überbietungsrolle argumentativ wirklich eingeholt würde. ${ }^{26}$

3. Als wahrhaft Lebendiger ist der eine Gott selber Geist, und seine Einheit lässt sich als eine kommunikative Einheit beschreiben, d. h. als von ihm selber her offen für die Gemeinschaft mit dem Anderen. Sie ist trinitarisch eine sich artikulierende Einheit - gemäß dem johanneitischen Wort: „Wie der Vater das Leben in sich selbst hat, so hat er auch dem Sohn gegeben, das Leben

ohne seinen (trinitarisch verstandenen) Geist gedacht, kommt es zu den problematischen Folgen eines abstrakten dominium terrae (vgl. 1Mose 1, 28: ,untertan machen“).

22 Sure 6, 101; 9, 30; 18, 3; 19, 91f(!) u. 36; 25, 2; 36, 9; 43, 81; 72, 3. Vgl. o. Anm. 8. Diese „Lüge“(18, 4) kann nur mit Totschlag beantwortet werden $(9,30)$. Ähnlich die wohl mehr gegen den paganen Polytheismus im alten Arabien gerichtete, häufige Mahnung, neben Allah keinen anderen Gott bzw. andere Götter zu setzen oder ihm „beizugesellen“ (Sure 2, 20; 3, 89 (vgl. 60); 16, 96; 17, 44; 25, 3; 36, 74; 58, 111). Umgekehrt wird die Dreieinigkeit nach dem Modell der „Beigesellung“ (sc. gewisser Mächte) aufgefasst.

23 Sure 4, 169; 5, 76.

24 Vgl. Sure 3, 138; 5, 79 mit 7, 59 u. 65.

25 Sure 33, 40; vgl. 9, 33; 12, 111.

26 Andeutungen in dieser Richtung bietet die (abstrakt bleibende) Behauptung von der „Bestätigung des Vorausgegangenen“ im Propheten (Sure 2, 85; 3, 2; 6, 92; 12, 111; 35, 28; 46, 29; vgl. 4, 161). 
zu haben in ihm selbst“ (Joh 5, 26). ${ }^{27}$ Gottes Lebendigkeit wird hier als sich mitteilende Liebe gedacht (vgl. Joh 3, 16).

Von da aus ist an den Koran die Frage zu richten: Bleibt nicht die ständige Prädizierung Allahs als des „Barmherzigen“ bzw. Allerbarmers unterbestimmt, weil nur auf einzelne kontingente Verfehlungen des Menschen bezogen? ${ }^{28}$ Weil hier die prinzipielle Notwendigkeit der Erlösung, die in einer grundlegenden Verkehrung des Gottesverhältnisses, in einer verkehrten menschlichen Selbstbezogenheit, d. h. der Sünde (im Singular!) gründet, unbekannt ist, kann nicht und braucht auch nicht eine grundlegende Versöhnung, die von Gott allein ausgehen kann, in Anschlag gebracht werden. Ohne kondeszendente Selbstmitteilung Gottes ist Versöhnung im eigentlichen Sinne nicht zu denken. Denn „zur Versöhnung kommt es nur dann, wenn der Eine nicht der geschlossene Einzige ist, ${ }^{29}$ wenn er [vielmehr] ohne Auflösung seiner Gottheit selbst der Vollzug von Gemeinschaft ist und sich auf die vielen so einlässt, dass er sich mit ihnen im Vollzug eines Lebens verbindet, um sie damit in die Gemeinschaft seiner eigenen [sc. innertrinitarischen] Gemeinschaftlichkeit aufzunehmen“ (J. Baur) ${ }^{30}$.

In dieser dreifachen Hinsicht: wahrer, lebendiger und kommunikativer Einheit ist der Glaube an den dreieinigen Gott konkreter Monotheismus.

Bleibt hingegen der unbewegte, schlechthin Eine und Einfache ${ }^{31}$ ein abstraktes Jenseits aller Vielheit, so kann der Mensch nur seinerseits durch eigene fromme Leistungen das Gottesverhältnis bestimmen. Dem entspricht das koranische Verständnis von „Glauben“, ${ }^{32}$ für den es übrigens im Arabischen kein echtes Äquivalent gibt ${ }^{33}$. Das so Benannte ist für den „Islam“ (= Ergebung) ${ }^{34}$ im Kern reine Unterwerfung unter das göttliche Wort Mohammeds des Gesandten und somit im formalen Sinne „Gehorsam“ (Sure 24, 50) ${ }^{35}$.

27 Entsprechend gewinnen auch die Glaubenden Anteil an diesem Leben Gottes selber (vgl. Joh 6, 53 u. 57).

28 Vgl. z. B. Sure 2, 35.

29 (Anm. J. R.) Hegel sprach vom „leblos Einsamen“ (Phänomenologie des Geistes, letzter Satz; Werke in zwanzig Bänden, 3, 591).

30 A.a.O., wie o. Anm. 5, 169.

31 Vgl. o. Anm. 4.

32 Vgl. Sure 4, 135; 24, 62; 45, 19; 98, 4.

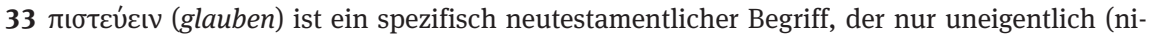
vellierend) z. B. in den Koran-Übersetzungen für den Islam gebraucht werden kann. Im Koran selber finden Ausdrücke wie din (Befolgung der Daseins- bzw. Schöpfungsordnung nach Allahs Normen) oder `ibada (rituelle Verehrung Allahs; Sure 51, 56) Verwendung. Vgl. auch u. Anm. 36. 34 Vgl. z. B. Sure 4, 124; 6, 70.

35 Dieser unter ständiger göttlicher Strafandrohung geforderte Gehorsam entspricht dem positiven Offenbarungsanspruch, der unmittelbar als faktische Gegebenheit für den Koran selber 
Abschließend und zusammenfassend ist zu sagen: Die Sachfrage nach dem Verhältnis von Islam und Christentum konzentriert sich zuletzt in der Frage nach dem Verständnis der Freiheit Gottes und wird konkret in der Frage nach der menschlichen Freiheit (subjektiv, gesellschaftlich, politisch und staatlich), sofern solche Freiheit allein im Gottesgedanken zu begründen is $t^{36}$.

geltend gemacht wird. Dem islam (von aslama: (sich) weggeben; muslim ist das Partizip dazu) entspricht wesentlich `ibada (s.o. Anm. 33) bzw. schabada (tätige Bezeugung der Einheit Allahs). 36 Der Status des frommen Muslim (zum Begriff vgl. die vorige Anm.) ist theologisch und scharia-rechtlich der einer „Knechtschaft“ gegenüber Allah ( $t a^{\prime}$ abbud). Vgl. dazu Joh 15, 12-15. 